\section{The incidence and} correlates of

non-adherence in adolescents receiving chemotherapy

by Jacqueline Ellis, Annette O'Connor, Jean Dunning, Laura Goodine, Dorothy Papineau and Brian Luke

\begin{abstract}
A retrospective chant review was conducted to investigate the prevalence of non-allherence to chemotherapy among adolescents with cancer.

Chemotherapy treatment protocols were identified as Treatment $I$ or Treatment II depending on treatment phase and disease progression. Treatment I was defined as the series of drugs given during the induction and maintenance phases of treatment. Treatment II was a different series of dnugs, offered if the patient failed to achieve remission after the induction phase or experienced relapse at any time duting treatment. Each treatment was analyzed separately and independently. Data were extracted from a total of 49 charts. Forty-eight charts had information available from the time of diagnosis and one chart had information only from the time of relapse because the patient received initial treatment at another centre.
\end{abstract}

Of the 48 patients who entered Treatment I, one patient refused chemotherapy and 47 accepted. The one patient who refused an initial offer of chemotherapy accepted an equivalent treatment of total nodal radiation. He was not considered non-adherent; however, factors that led to his decision to reficse chemotherapy will be examined.

Of the 47 patients who received chemotherapy in Treatment $I$, one patient dropped out, four patients modified their treament and five delayed treatment, for a total non-adherence rate of $21 \%(n=10)$. There were a total of 16 patients in Treatment II which was composed of 15 of the initial 47 patients in Treatment $I$ and one patient transferred from another hospital. Of the 16 patients in Treatment II, wo patients dropped out and three patients modified treatment for a non-adherence rate of $31 \%(n=5)$.

The major reason for modification or delay of treatment was the inabiliny to tolerate nausea and vaniting. Antiemetics were refused by 13 patients $(27 \%)$ due to the umpleasant side effects of these dnugs. Other factors contributing to non-adherence and implications of this shudy for future research are discussed.

\section{Introduction}

At the time of diagnosis, adolescent cancer patients and their families receive information about the nature of their illness and are advised of treatment protocols which will improve their chances of long-term survival. Although the protocol improves the prognosis, it results in unpleasant side effects, inconvenience, and restrictions in activities of daily living. Often, decisions about following protocols involve making trade-offs between quality of life and longevity. For many patients, or their families, the decision is straightforward; survival takes primacy in decision-making and quality-of-life factors are secondary. For others, the treatment is considered worse than the disease. Either in anticipation of the toxicity and complications, or after experiencing them for a period of time, patients may decide to drop out of treatment, or have treatment modified because the restrictions imposed on their quality of life outweigh the possible benefit of reduced morbidity and mortality.

The purpose of this study was to examine the prevalence and factors associated with non-adherence to chemotherapy in a group of adolescents. Through a retrospective chart audit, decisions made by the adolescent to refuse, modify, delay, or discontinue treatment were identified and factors contributing to these decisions were noted. The chart audit was chosen as a means to give direction to a more extensive, prospective study that would examine, in more detail, patient decision-making about non-adherence.

The prevalence of non-adherence across a variety of conditions in adult and paediatric populations has been shown to be significant. Approximately $50 \%$ of all patients, regardless of age or condition, have difficulty following prescribed medical regimens (Sackett, \& Snow, 1979). In a review of 11 adherencestudies with chronically ill children, Litt and Cuskey (1980) found non-adherence rates ranging from 12 to $\& 8$ per cent. When adolescents were separated out, they were found to be less adherent than younger children. Factors, regardless of age, that have been found to adversely affect adherence include:

(1) the length and complexity of the prescribed regimen (Haynes, Taylor, \& Sackett, 1979),

(2) side effects (Korsh, Fine, \& Negrete, 1978; Smith, Rasen, Trueworthy, \& Lowman, 1979),

(3) the degree of difficulty in managing side effects (Richardson, Marks \& Levine, 1988) and,

(4) the degree to which the child's normal developmental function is interrupted (Koocher 1986; Friedman et al, 1986).

The complex nature of cancer treatment protocols may make them particularly difficult to follow. Adherence, for the adolescent, means time

\section{L'INCIDENCE ET LES CORRÉLATIFS DE LA NON-OBSERVATION CHEZ LES ADOLESCENTS TRAITÉS PAR CHIMIOTHÉRAPIE}

\section{ABRÉGÉ}

Un examen rétrospectif des dossiers médicaux a été mené pour étudier la prévalence de la non-observation de la chimiothérapie chez les adolescents atteints du cancer. Les protocoles de traitement de chimiothérapie ont été identifiés sous le nom de Traitement I ou Traitement II en fonction de la phase de traitement et de progression de la maladie. Le Traitement I correspond à la série de médicaments donnée pendant les phases d'amorçage et de maintien du traitement. Le Traitement II correspond à une différente série de médicaments donnée au malade s'il n'y pas eu rémission après la phase d'amorçage ou si la maladie recommence à progresser à n'importe quel moment du traitement. Chaque traitement a été fait l'objet d'une étude séparée et indépendante. Nous avons extrait des données de 49 dossiers. Quarante-huit de ces dossiers portaient des renseignements qui remontaient au diagnostic et le dossier restant ne commençait qu'à partir de la rechute étant donné que le patient avait reçu son traitement initial dans un autre centre.

Jacqueline Ellis, RN, MScN, Annette O'Connor, RN, $\mathrm{PhD}$, and Jean Dunning, RN, MScN, are with the Faculty of Health Sciences, University of Ottawa. Laura Goodine is a doctoral student at Carleton University. Dorothy Papineau, RN and Brian Luke, MD, FRCP, are with the Haematology/Oncology Service at Children's Hospital of Eastern Ontario in Ottawa. 
spent in hospital away from family, school and peers; numerous clinic visits; painful diagnostic and evaluation procedures and living with the toxic effects of the chemotherapy drugs. Drug side effects that are visible such as weight gain, acne, and hair loss are most disturbing to adolescents (Klopovich, \& Trueworthy 1985) as this altered appearance conflicts with their need to conform to the image of their peers. At a time when peer acoeptance, body image and emerging independence are important issues for the adolescent, cancer treatment forces them to be more dependent on their parents and sets them visibly apart from their peers.

Drug therapy for the treatment of cancer is considered to be instrumental in achieving disease-free survival (Pinkel 1976), yet little detailed information is available about the consequences of delay, dosage modification, or refusal of drugs within a protocol. Most clinicians would agree that labelling an individual as non-adherent has negative implications, however this must be weighed against the benefits of having targeted an individual for added support and intervention that might help them to comply with therapy.

Varying definitions of what behaviours constitute adherence, and diverse techniques for measuring adherence make it problematic to compare results across studies. Indirect measures such as pill counts, appointment keeping, prescription renewal rates and patient testimony do not always give an accurate picture of patient adherence (Sackett, \& Snow 1979). Direct methods involving serum drug levels or urine metabolites are more accurate but are expensive and can involve invasive procedures. Depending on the extent to which adherence was operationally defined and reliably measured, study results must be interpreted with caution.

Smith, Rosen, Trueworthy, \& Lowman (1979) were the first investigators to use a direct measure of medication adherence. Adherence to prednisone therapy was determined using a random, urine 17-ketogenic steroid assay. Thirty-three per cent of the total assays performed were below the value set for adherence and of the 17 assays performed on eight patients over 11 years, $59 \%$ were in the non-adherence range.

Lansky, Smith, Cairns, N. and Cairns, G. (1983) used the same method to measure prednisone adherence in 31 leukemic patients under 15 years of age. The over-all non-adherence rate was $42 \%$ with no patient found to be $100 \%$ adherent. While both of these studies used a rigorous method for determining adherence, they are limited to prednisone therapy which is only a part of the treatment for cancer. It is speculative to generalize adherence behaviour with other drugs in the protocol based on the adolescent's adherence to prednisone therapy.

Tebbi, Cummings, Zevon, Smith, Richards, \& Mallon (1986) measured self-reported non-adherence with prednisone and corroborated the testimony with serum bioassays. They found that children over 10 years of age had a greater adherence problem than younger children. An interesting finding from this study was the observation that patients/parents gave accurate acoounts of missed dosages which were supported by the results of the serum bioassays. A limitation of the study was the definition of non-adherence which included patients missing from one to greater than three doses. In terms of the impact on survival, missing one dose of a drug probably does not have the same implications as missing many doses or taking the drugs sporadically. It seems unrealistic, given the complexity and prolonged length of cancer treatment protocok, to classify patients as non-adherent if they miss one dose of medication.

In studies that used indirect methods to measure adherence, such as physicians' perceptions (Dolgin, Katz, Doctors, \& Siegel, 1986), clinic attendance (Levanthal, \& Boek, 1978; MacDougall, Wilson, Cohn, Shuenyane, \& McElligott, 1989), and patient self-report (Macdougall et al, 1989), non-adherence was a significant problem, with adolescents generally less adherent than younger children.

Two studies have established a link between prednisone adherence and survival. Trueworthy (1982) categorized 17 children with lymphoblastic leukemia as adherent or non-adherent based on urinary assays. The 12 patients in the adherent group had no incidence of relapse whereas four of the five patients in the non-adherent group had relapsed. Richardson, Shelton, Krailo and Levine (1990) used serum drug and metabolite levels to assess adherence to allopurinal and prednisone in 94 adults. They found a significant correlation between adherence with oral medication and survival.

The results of adherence research suggests that adolescents have a difficult time following treatment protocols. However, it is difficult to know the exact scope of the problem and the implications for survival. In order to be meaningful, the problem of adherence needs to be studied from a broader perspective, taking into account different phases of treatment eg, induction, maintenance, after relapse, and examining provider and self-administered chemotherapy. Most of the studies focused on one drug and none mentioned at what point in treatment the non-adherence behaviour occurred. Non-adherence during induction may have different implications for survival than non-adherence after relapse. This study was conducted to examine the incidence and factors associated with treatment refusal, delay, modification or discontinuation of provider-administered chemotherapy during induction and maintenance (Treatment I) and after relapse (Treatment II).

\section{Method}

\section{Procedure}

A retrospective chart review was conducted at a children's hospital in Ontario. The centre cares for approximately 40 newly diagnosed cancer patients per year and has a total active caseload of 200 patients per year. Chart audits were conducted to examine treatment decisions for all adolescents offered chemotherapy over a ten-year period (1979-1988). Individuals ranging in age from 11 to 18 years were classified as adolescents. This age span covers early, middle and late adolescence as described by Felice (1983). All diagnostic categories were considered eligible to be included in the study. Treatment protocols were classified as Treatment I or Treatment II depending on whether the patient entered at the time of diagnosis (I) or at treatment failure/relapse (II).

Charts of all patients who were offered chemotherapy over a 10-year period were identified using the Canadian Classification of Diagnostic, Therapeutic and Surgical Procedures code for injection or infusion of cancer chemotherapy substances. Of the 231 charts screened for inclusion in the study, 49 were between the ages of 11 and 18 , and were no longer receiving chemotherapy. Forty-eight charts selected were from patients treated at the time of diagnosis (Treatment I) and one chart was from a patient transferred to the centre at the time of relapse (Treatment II), having finished the initial treatment elsewhere (sec Figure One). Information concerning chemotherapy adherence was extracted from medication and treatment records, physicians', nurses' and allied health workers' notes. The notes for each clinic visit were examined to ascertain if the adolescent received the treatment that was plannedat the previous visit. For example, if the physician's note read "return to clinic in one week for Vincristine $2 \mathrm{mg} \mathrm{IV"} \mathrm{it} \mathrm{was} \mathrm{then}$ possible to evaluate the next visit to see if the patient kept the appointment and received the drug as planned. If the drug was not given or the dosage was modified the reasons were classified as patient or physician-determined. Physician-determined reasons were mainly related to drug toxicities such as decreased white blood count, infection, stomatitis, diarrhea etc. Examples of patient-determined reasons for modifying or delaying chemotherapy were

Figure One: Flow Diagram of Patients

Charts of 49 patients reviewed

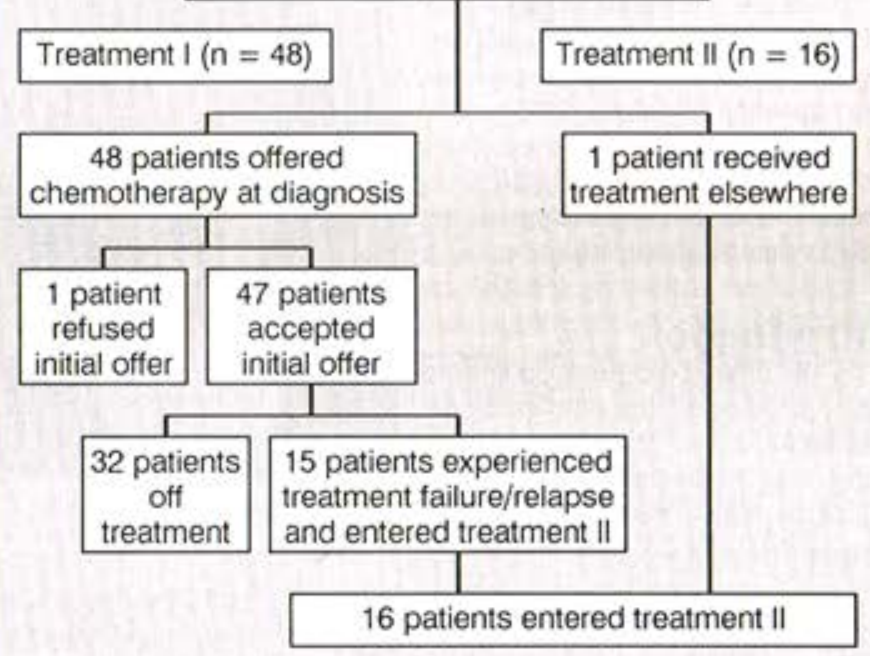


cancelled appointments, pain, nausea, refusal etc. We did not attempt to analyze how much, or what type of input parents had into initiating changes in treatment because, for the most part, it was not possible to obtain this information retrospectively from the charts.

Results of each chart review were validated with the principle investigator and two research assistants. The nurse in the oncology clinic was consulted to clarify information in the charts when needed. If the patient entered more than one treatment protocol, which was the case for all patients that relapsed, each treatment was analyzed separately. Rates of non-adherence are reported for Treatment $I$, which is the series of chemotherapy drugs given for the induction and maintenance phases of treatment, and Treatment II, which begins at the time of relapse or failure of Treatment I to put the patient into remission. These treatments were analyzed separately and independently of each other. Other variables extracted from the medical record included: Diagnosis, age at diagnosis, sex, type of treatment protocol, types of health professionals involved with the patient, and side effects related to chemotherapy,

Analysis

Rates of adherenceand non-adherence were calculated based on patients decisions. Three decision categories were established. Adolescents that delayed or modified treatment on three or more occasions or patients that refused/dropped out of treatment were considered non-adherent. In an effort to recognize that some flexibility exists within treatment protocols, adherence was defined as less than three episodes of chemotherapy delay or modification. Due to the lengthy and complex nature of cancer treatment

Figure Two: Adherence by age and sex in Treatment 1

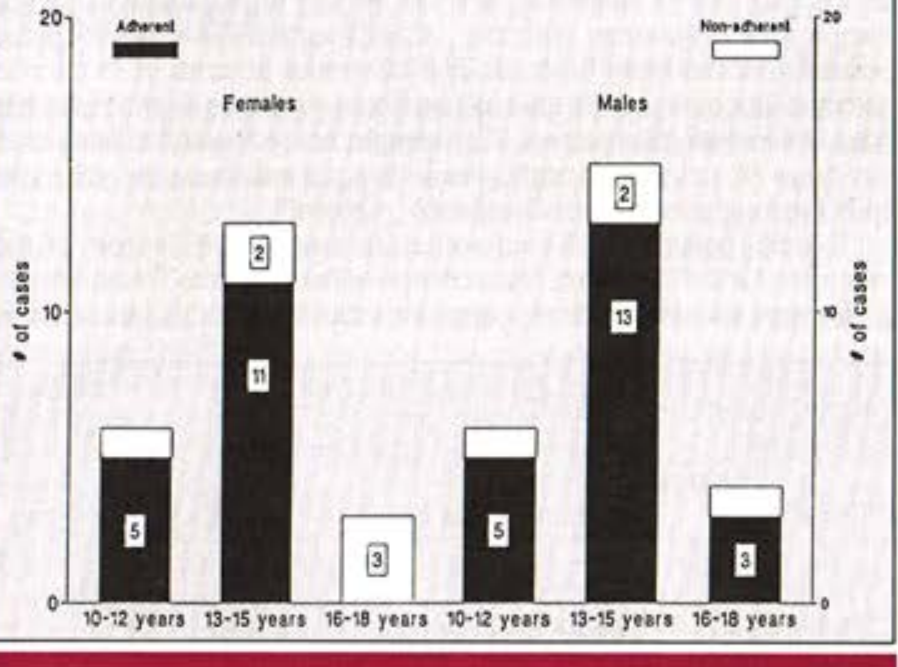

Figure Three: Adherence by diagnosis in Treatment I

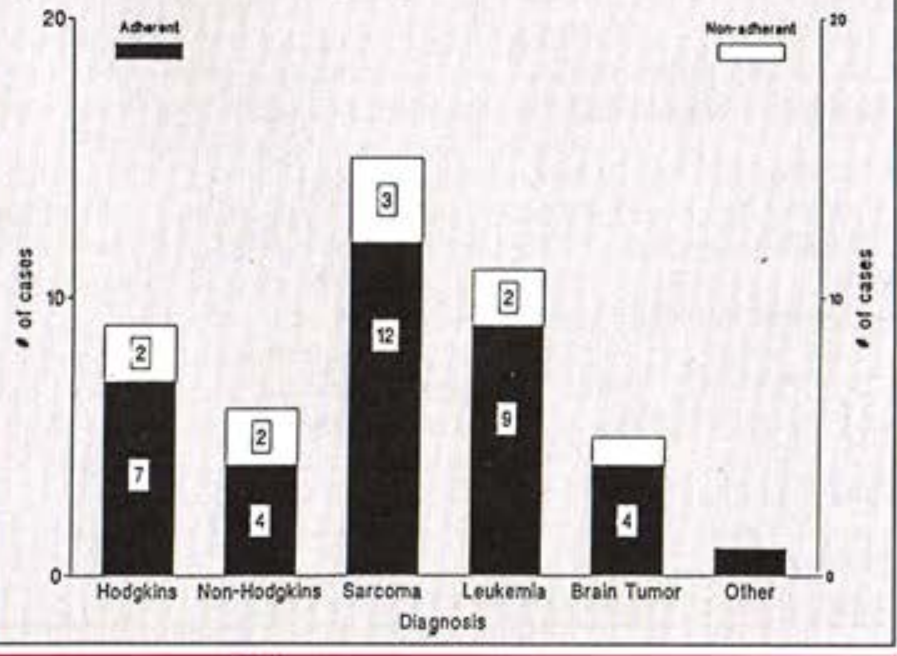

protocols, it did not seem realistic to classify an adolescent as non-adherent if there were infrequent modifications or delays in chemotherapy administration. Three episodes was arbitrarily chosen as a reasonable dividing line between adherence and non-adherence. There is nothing in the literature to provide guidelines as to how much a protocol can be altered before treatment success or survival is affected.

Descriptive statistics were used to describe the sample. Chisquare analysis and Fisher's exact test were used to test the relationship between demographic and clinical variables and adherence categories.

Results

The 49 patients selected for inclusion in the study ranged in age at time of diagnosis from 11.5 to 18 years with a mean age of 13.82 (SD 1.7). Twenty-eight ( $57 \%$ ) patients were 13 to 15 years of age. There were 25 males and 24 females in the study. Lymphoma was the largest diagnostic category with $16(33 \%)$ patients. The second largest category was sarcoma with 15 $(31 \%)$ patients. Outcome data indicated that $30(61 \%)$ of the patients were alive at the time of the chart review and $19(39 \%)$ of the patients had died. Figure Two shows adherence status in Treatment I byage and sex, and Figure Three shows adherence status by diagnosis.

The modal patient in this sample was male, diagnosed at 12 years of age with a diagnosis of lymphoma or sarcoma. He followed only one protocol (Treatment I), a T10 or a MOPP(Devita, Serpick, \& Carbone 1970) and was alive at outcome. In addition to nurses and physicians, he had a social worker and a child life worker involved in his care.

Among those patients entering Treatment I, the non-adherence rate was $21 \%$, with a $95 \%$ confidence interval of 9 to 33 . This non-adherence rate included nine modifier/delayers, and one drop-out. Although the non-adherence rate for Treatment II was $31 \%$, it was not considered meaningful due to the small number of patients $(n=16)$ and the resultant wide confidence intervals.

It was noted that $13(27 \%)$ patients refused IV and oral antiemetics sometime during their treatment. Refusal was linked to the side-effects of the antiemetics. Statements such as "they make me feel out of control", or "I don't like the way they make me feel", were typical of patients' reasons for refusing the antiemetics. The relationship between adherence status and refusal of antiemetics is presented in Figure Four.

Refuser/drop-out rates

Of the 48 patients offered chemotherapy at the time of diagnosis (Treatment I), one patient refused, and one patient dropped out of treatment after completing 12 months of an 18-month protocol. The patient who refused chemotherapy was male, 17 years of age at the time of diagnosis, with Stage II, Hodgkin's lymphoma. In conjunction with the oncologist and his parents, he opted for total nodal radiation (Stanford protocol) with the understanding that he might have to undergo chemotherapy if radiotherapy proved inadequate. He was not entered into the statistical analysis as non-adherent because be acoepted an alternative to chemotherapy that did not jeopardize his chances of survival. Reasons given for refusal of

Figure Four: Refusal of antiemetics by adherence status across Treatments I and II

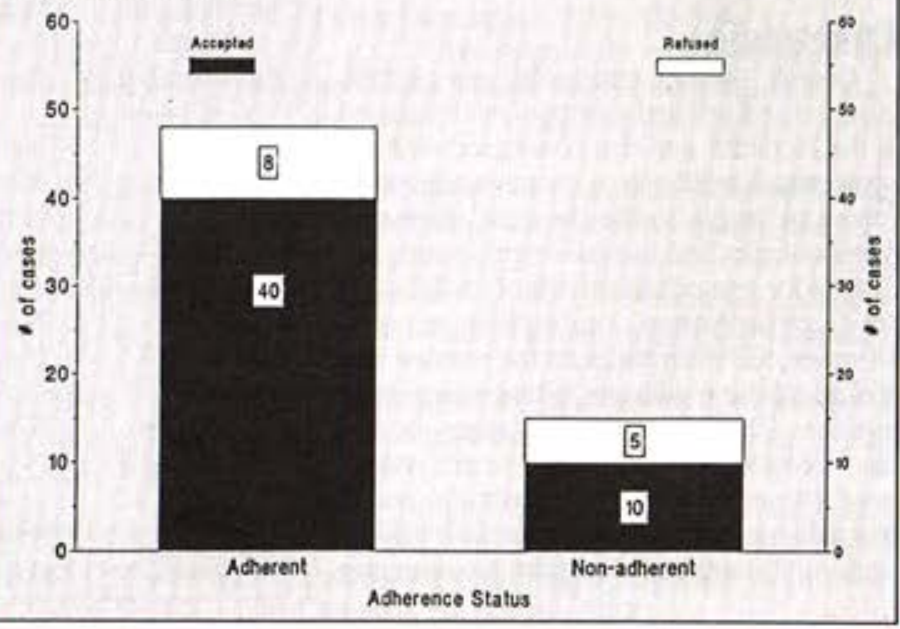


chemotherapy were related to hair loss and the fear of becoming sterile as a result of chemotherapy.

The patient who dropped out of treatment was also male, and diagnosed at 12 years with non-Hodgkin's lymphoma. He was noted to have refused chemotherapy due to vomiting and/or fear of needles at least nine times before finally dropping out of treatment. Currently he is alive and in remission.

Sixteen of the 49 patients entered Treatment II; of this group, two $(10 \%)$ patients dropped out. Both were males, one diagnosed at 13 years with malignant schwannoma and the other diagnosed at 16 years with Ewing's sarcoma of the right wrist. Both patients were adherent in Treatment I, relapsed and were offered a second series of drugs (Treatment II). The patient with malignant schwannoma refused chemotherapy, opted for radiotherapy and subsequently died. The patient with Ewing's sarcoma indicated many times that he did not want any more chemotherapy before finally choosing not to have further treatment. He did return for chemotherapy at another centre for a short time before his death.

\section{Delay/modification rates}

In Treatment $I(n=47)$, four patients were categorized as modifiers and five patients were categorized as delayers for a combined rate of $19 \%$. In Treatment II $(\mathrm{n}=16)$, two patients delayed treatment and one patient modified treatment for a delay/modification rate of $19 \%$.

The most frequently cited explanations for non-adherence were nausea and vomiting, missed appointments, pain and a nonspecific complaint of "feeling unwell." Overall, the reasons for non-adherence can be categorized into drug side-effects, psychological and social variables. Drug side-effects that contributed to non-adherence included pain, nausea and vomiting, loss of appetite, dizziness, and generally feeling "unwell". The psychological factors most frequently cited in the charts were depression, fatigue/tension, fear of hair loss, anxiety, and fear of needles. The social variables that contributed to treatment delay or modification included family vacations, school-related activities, cancelled appointments and one instance of medication being withheld by the mother, as a punishment.

Correlates of non-adherence

Several variables had more than $25 \%$ of the cells with expected frequencies less than five. Therefore Fisher's exact test was used to replace Chi square analyses where appropriate. Adherence was considered in view of its possible association with age, sex, diagnosis, outcome, treatment regimen and number of health professionals involved other than nurses or physicians. (See Table One) The significance level was adjusted to $\mathrm{p}<.008$ to account for the increased chance of obtaining a type I error. There was no statistically significant association between adherence status and any of the demographic, diagnostic and treatment variables. The lack of statistical correlation between selected variables and adherence may be due to small sample sizes. In at least three variables (sex, diagnosis and outcome) absolute differences in proportions of $15 \%$ were noted.

\section{Discussion}

Overall, the non-adherence rate of $21 \%$ appears to fallwithin the range of rates reported in the literature and was considered clinically significant. It is difficult to compare adherence behaviour across studies because of diverse methodologies and measurement techniques. Criteria for non-adherence are often different, not clearly articulated, or subjectively reported. However, this study supports the premise that adolescents have difficulty following treatment regimens. This is not surprising given the fact that adolescents are at an age where control over their lives is an important issue. Non-adherence behaviour becomes a vehicle, albeit maladaptive, to exercise an emerging sense of power and autonomy within the structured, often inflexible routines of hospitals and clinics.

A limitation of this study was the fact that it was a retrospective chart review. The amount of information in the chart, the logic and clarity of the notes tended to vary depending on the author. However, it was still possible to get a sense of how well or poorly the patient was coping with treatment. When drugs were refused or dosages modified there was a notation on the chart giving some explanation. It was then possible toclassify the modifications or delays as patient or physician-directed. It was also apparent that many of the adherent patients had great difficulty coping with treatment side-effects, school absences, and the overall stress of having cancer.

The development of new treatments and the success of existing treatment protocols have dramatically increased long-term survival rates for adolescents with cancer. However, the efficacy of a treatment protocol is, in part, dependent on howwell the patient is able to followit. Therefore, it is important to be able to predict which adolescents may not comply with treatment and which treatments are particularly difficult to follow. Predictor variables could be identified and tested in a prospective study. Patients identified as being at risk for non-adherence could be targeted for intervention before a problem develops. Variables identified from this study which may be useful to include in a prospective study are severity of nausea and vomiting, refusal of antiemetics, and support provided by family, and by health professionals.

The refuser/dropout rate of $2 \%$, in Treatment $I$, was composed of one patient who dropped out before treatment was completed. This is in contrast to the $19 \%(\mathrm{n}=9)$ of patients in Treatment I who delayed or modified their treatment regimens when no objective toxicity or treatment failure was noted. Although modification and delay of treatment is not as extreme as refusing or dropping out of treatment, it is not known to what extent this may compromise treatment outcome. A prospective study would permit evaluation of this more moderate non-adherence in terms of its effect on outcome. It would provide information about the extent to which flexibility exists within a specific protocol. For example, how often and to what extent can drugs be delayed or omitted and dosages modified, and still expect optimum long-term survival rates from a protocol?

Thirteen patients $(27 \%)$ refused antiemetics for all or part of their treatment. Of the 15 patients that were non-adherent across Treatment Iand II, five were also antiemetic refusers, for a total of $33 \%$ of the non-adherent

Table One: Correlates of adherence in treatment $\mathrm{I}(\mathrm{N}=47)$

\begin{tabular}{|c|c|c|c|c|c|}
\hline Variable & \multicolumn{2}{|c|}{ Adherent $(\mathrm{N}=37)$} & $\begin{array}{l}\text { Non-adherent }(\mathrm{N}=10) \\
\text { Status }\end{array}$ & $\mathrm{X}^{2}$ & PValue \\
\hline \multicolumn{6}{|c|}{ Age at diagnosis } \\
\hline$<14$ & $\mathrm{~N}=21$ & $17(46 \%)$ & $4(40 \%)$ & $* *$ & 1.00 \\
\hline$\geq 14$ & $\mathrm{~N}=26$ & $20(54 \%)$ & $6(60 \%)$ & & \\
\hline \multicolumn{6}{|l|}{ Sex } \\
\hline Male & $\mathrm{N}=25$ & $21(57 \%)$ & $4(40 \%)$ & & \\
\hline Female & $\mathrm{N}=22$ & $16(43 \%)$ & $6(60 \%)$ & .17 & .68 \\
\hline \multicolumn{6}{|l|}{ Diagnosis* } \\
\hline Lymphoma & $\mathrm{N}=15$ & $10(27 \%)$ & $5(50 \%)$ & & \\
\hline Sarcoma & $\mathrm{N}=15$ & $12(32 \%)$ & $3(30 \%)$ & 1.04 & .60 \\
\hline Leukemia & $\mathrm{N}=11$ & $9(24 \%)$ & $2(20 \%)$ & & \\
\hline \multicolumn{6}{|c|}{ Type of protocol } \\
\hline MOPP & $N=9$ & $7(19 \%)$ & $2(20 \%)$ & & \\
\hline T-9 & $N=8$ & $7(19 \%)$ & $1(10 \%)$ & .45 & 80 \\
\hline Others & $\mathrm{N}=30$ & $23(62 \%)$ & $7(70 \%)$ & & \\
\hline \multirow{2}{*}{\multicolumn{6}{|c|}{$\begin{array}{l}\text { Number of health professionals } \\
\text { involved in addition to physicians and nurses }\end{array}$}} \\
\hline & & & & & \\
\hline$<2$ & $\mathrm{~N}=17$ & $13(35 \%)$ & $4(45 \%)$ & & \\
\hline$\geq 2$ & $\mathrm{~N}=30$ & $24(65 \%)$ & $6(35 \%)$ & $* *$ & 1.00 \\
\hline \multicolumn{6}{|l|}{ Outcome } \\
\hline Alive & $\mathrm{N}=30$ & $22(59 \%)$ & $8(75 \%)$ & & \\
\hline Dead & $\mathrm{N}=17$ & $15(41 \%)$ & $2(25 \%)$ & & .29 \\
\hline
\end{tabular}

* These were the three largest categories

** Fisher's exact test used 
group. A few patients made specific statements about side-effects of the antiemetics that they found intolerable. Because one of the major reasons for non-adherenoe was nausea and vomiting, it would be useful to determine factors contributing to antiemetic refusal and how this affects adherence.

The difficulties that adolescents have coping with the side-effects of antiemetic therapy suggest the need for drugs that can control nausea and vomiting without the drowsiness, extrapyramidal effects and perceptual alterations that are common with current therapy. Ondansetron hydrochloride (Zofran), a promising new antiemetic, seems to be very effective in controlling nausea and vomiting without undesirable side-effects (Marty, Pouillart, School, et al 1990; Cubeddu, Hoffman, Fuenmayor, \& Finn 1990; Schmoll, 1989). At this point, research with ondansetron is limited, due to its relatively recent development. It would be useful to evaluate how acoeptable this drug is to the adolescents and what impact alleviation of nausea and vomiting has on adherence.

In addition to drug therapy, it would be interesting to look at alternative approaches to the treatment of chemotherapy-induced nausea and vomiting and subsequent effects on adherence. Some sucoess in alleviating post treatment and anticipatory nausea and vomiting has been reported with relaxation techniques such as guided imagery, hypnosis (Cotanch, Hockenberry \& Herman, 1985), systematic desensitization (Morrow \& Morrell, 1982), progressive muscle relaxation and biofeedback (Burish, Shartner \& Lyles, 1981). These techniques are relatively easy to learn, can be controlled by the patient, and have no side-effects. They might prove especially useful for adolescents who refuse antiemetic drugs.

\section{References}

1. Burish, T.,Shartner, C., Lyles, J. Effectiveness of multiple muscle site EMG biofeedback and relaxation training in reducing adverseness of cancer chemotherapy. Biofeed Self Regul, 6, 1981, 523-35.

2. Cotanch, P., Hockenberry, M., Herman, S. Self-hypnosis as antiemetic therapy in children receiving chemotherapy. Oncology Nursing Forum, 12, $1985,41-46$.

3. Cubeddu, L.X., Hoffman, I.S., Fuenmayor, N.T., Finn, A.L. Efficacy of ondansetron (GR38032F) and the role of serotonin in cisplatin-induced nausea and vomiting. New England Journal of Medicine, 322, 1990, 810-816. 4. Devita, V.T., Serpick, A.A., Carbone, P.P. Combination chemotherapy in the treatment of advanced Hodgkin's disease. Annals of Internal Medicine, 73, 1970, 881-895.

5. Dolgin, M.J., Katz, E.R., Doctors, S.R., Seigel, S.E. Caregivers' perceptions of medical compliance in adolescents with cancer. Journal of Adolescent Health Care, 7, 1986, 22-27.

6. Felice, M.E.,Adolescence: General considerations. In Levine, M.D., Carey, W.B., Crocker, A.C., Gross, R.T. (eds) Developmental-behavioral pediatrics. Philadelphia: W.B. Saunders, 1983, 133-149,

7. Friedman, I.M., Litt, I.F., King, D.R., Henson, R., Holtzman, D., Halverson, D., Kraemer, H.C. Compliance with anticonvulsant therapy by epileptic youth: Relationships to psychosocial aspects of adolescent development. Journal of Adolescent Health Care, 7, 1986, 12-17.

8. Haynes, R.B., Taylor, D.W., Sackett, D.L. (eds) Compliance in Health Care. Baltimore: Johns Hopkins University Press, 1979.

9. Klopovich, P.M., Trueworthy, R.C. Adherence to chemotherapy regimens among children with cancer. Topics in Clinical Nursing, April, 1985, 19-25. 10. Koocher, G.P. Psychosocial issues during the acute treatment of pediatric cancer. Cancer, 58, 1986, 468-472.

11. Korsh, B.M., Fine, R.N., Negrete, V.F. Noncompliance in children with renal transplants. Pediatrics, 6, 1978, 872-876.

12. Lansky, S.B., Smith, S.D., Cairns, N.U., Cairns, G.F. Psychological correlates of compliance. The American Journal of Pediatric Hematology, 5, 1983, 87-92.

13. Leventhal, B.G., Boek. The adolescent with cancer. Proceedings of the American Cancer Society's 2nd National Conference on Human Valuesand Cancer, 1978.

14. Litt, I.F., Cuskey, W.R. Compliance with medical regimens during adolescence. Pediatric Clinies of North America, 27, (2), 1980, 3-15.
The extent to which social support influences adherence is an area that could use further study. There was anecdotal evidence in the charts indicating many of the patients who modified and delayed treatment also had chaotic family relationships. In one instance, a mother failed to renew her daughter's prescription for chemotherapy for three weeks as a punishment for bad behaviour. In other instances, it was mentioned that parents were having difficulties coping with their own lives and were not able tooffer much support to the patient. It is not known to what degree family stability or support is influential in adherence in an adolescent cancer population, nor towhat extent health care professionals can aid in a family situation where social support is lacking. Further investigation may provide more understanding in this area.

\section{Summary}

In summary, non-adherence is a complex phenomenon and, in adolescents, one we know very little about. The significant rate of non-adherence observed in this Canadian adolescent population warrants a prospective study. Patient interviews would give us information about the specific factors that lead to the decision to delay or modify treatment. Treatment delay and modification needs to be examined as it relates to long-term survival. The severity and persistence of nausea and vomiting and the inability to tolerate antiemetics need to be examined in relation to treatment delay and modification.

\section{Acknowledgement}

This study was funded by the Leukemia Research Fund, Tononto, Ontario. Special thanks to Jane Simpson $R N$, research assistant.
15. MacDougall, L_G., Wilson, T.D., Cohn, R., Shuenyane, E.N., McElligott, S.E. Compliance with chemotherapy in childhood leukaemia in Africa. South African Medical Journal, 75, 1989, 481-484.

16. Martin, N.J. Patients' decision making regarding intravenous chemotherapy for breast cancer or Hodgkin's lymphoma. Unpublished Masters Thesis. University of Toronto, Ontario, 1990.

17. Marty, M., Pouillart, P., Scholl, S., Droz, J.P., Azab, M., Brion, N. et al. Comparison of the 5-hydroxytryptamine (serotonin) antagonist ondansetron (GR38032G) with high-dose metoclopramide in the control of cisplatin-induced emesis. The New England Journal of Medicine, 322, 1990, 816-821.

18. Morrow, G., Morell, C. Behavioral treatment for the anticipatory nausea and vomiting induced by cancer chemotherapy. New England Journal of Medicine, 307, 1982, 1476-80.

19. Pinkel, D. Curability of childhood cancer. JAMA, 235, 1976, 1049-1050. 20. Richardson, J.L., Marks, G., Levine, A. The influence of symptoms of disease and side effects of treatment on compliance with cancer therapy. Journal of Clinical Oncology, 6, 1988, 1746-1752

21. Richardson, J.L., Shelton, D.R., Krailo, M., Levine, A. The effect of compliance with treatment on survival among patients with haematologic malignancies. Journal of Clinical Oncology, 8, 1990, 356-364.

22. Sackett, D.L., Snow, J.C. The magnitude of compliance and noncompliance. In Haynes, R.B., Taylor, D.W., Sackett, D.L. (eds) Compliance in Health Care. Baltimore: Johns Hopkins University Press, $1979,11-22$.

23. Schmoll, HJ. The role of ondansetron in the treatment of emesis induced by non-cisplatin-containing chemotherapy regimens. European Journal of Clinical Oncology, 25, (Supl. 1), 1989, 35-39.

24. Smith, D.D., Rosen, D., Trueworthy, R.C., Lowman, J.T. A reliable method for evaluating drug compliance in children with cancer. Cancer, 43 , $1979,169-173$.

25. Tebbi, C.K., Cummings, K.M., Zevon, M.A., Smith, L, Richards, M., Mallon, J. Compliance of pediatric and adolescent cancer patients. Cancer, $58,1986,1179-1186$.

26. Trueworthy, R.C. A new prognostic factor for childhood acute lymphoblastic leukemia: Drug absorption and compliance. Proceedings of the 4th annual Pediatric Haematology/Oncology Symposium, at University of Kansas Medical Center, Kansas City, Kansas, 1982. 